\title{
Editorial
}

\section{The TIDieR checklist will benefit the physiotherapy profession}

\author{
Tiê Yamato ${ }^{\mathrm{a}, *}$, Chris Maher $^{\mathrm{a}}$, Bruno Saragiotto $^{\mathrm{a}}$, Anne Moseley $^{\mathrm{a}}$, Tammy Hoffmann $^{\mathrm{b}}$, Mark Elkins ${ }^{\mathrm{c}}$ \\ and Joseph G. McVeigh ${ }^{\mathrm{d}}$ \\ ${ }^{a}$ Musculoskeletal Division, The George Institute for Global Health, Sydney Medical School, \\ The University of Sydney, Sydney, NSW, Australia \\ ${ }^{\mathrm{b}}$ Centre for Research in Evidence Based Practice, Faculty of Health Sciences and Medicine, \\ Bond University, Queensland, Australia \\ ${ }^{\mathrm{c}}$ International Society of Physiotherapy Journal Editors \\ ${ }^{\mathrm{d}}$ Institute of Nursing and Health Research, School of Health Sciences, Ulster University, Jordanstown, UK
}

Evidence-based practice involves physiotherapists incorporating high-quality clinical research on treatment efficacy into their clinical decision-making [1]. However, if clinical interventions are not adequately reported in the literature, physiotherapists face an important barrier to using effective interventions for their patients. Previous studies have reported that incomplete description of interventions is a problem in reports of randomised controlled trials in many health areas [2-4]. One of these studies [4] examined 133 trials of non-pharmacological interventions. The experimental intervention was inadequately described in over $60 \%$ of the trials and descriptions of the control interventions were even worse.

A recent study [5] evaluated the completeness of descriptions of the physiotherapy interventions in a sample of 200 randomised controlled trials published in 2013. Overall, the interventions were poorly described. For the intervention groups, about onequarter of the trials did not fulfil at least half of the

*Corresponding author: Tiê Yamato, The George Institute for Global Health, PO Box M201, Missenden Road, NSW 2050, Australia. E-mail: tyamato@georgeinstitute.org.au. criteria. Reporting for the control groups was even worse, with around three-quarters of trials not fulfilling at least half of the criteria. In other words, for the majority of the physiotherapy trials, clinicians and researchers would be unable to replicate the interventions that were tested.

Describing a treatment may seem like a simple task but physiotherapy interventions can be very complex. Some interventions are multi-modal, involving the use of manual techniques, consumable materials, equipment, education, training and feedback. Some interventions are tailored to each patient's specific health state, including the patient's immediate response to the application of the treatment. When the intervention involves a course of treatments, the intensity or dose may be progressed over time. The descriptions of physiotherapy interventions in trial reports often do not capture all these components of the interventions or detail their complexity.

If researchers fail to comprehensively report all aspects of the interventions, the trial results cannot be incorporated into clinical practice or the intervention could be implemented incorrectly. Incorrect implementation may make the treatment ineffective, 
wasting the clinician's and patient's time and healthcare resources. Inadequate reporting of interventions also poses a barrier to incorporating a trial's results into secondary research such as systematic reviews and clinical practice guidelines, as well as the usability of these resources. This means that the resources that were invested in undertaking the trial have been wasted. Such resources are extensive, including direct trial costs (e.g., payment of researchers, consumables), use of infrastructure (e.g., clinic space, equipment), human resources (e.g., ethics committee review, granting body review) and the goodwill of patients who agree to participate. Currently, there is a growing realisation that we need strategies to reduce waste in clinical research [6]. When the list of resources involved in a single study is considered, improving the reproducibility of interventions through better reporting could markedly reduce waste in research [7].

The TIDieR checklist and guide were developed to improve the reporting of interventions in any evaluative study, including randomised trials [8]. The checklist contains 12 items and was developed as an extension to the CONSORT 2010 [9] and SPIRIT 2013 [10] statements to provide further guidance for authors on the key information to include in trial reports. TIDieR items include: name of the intervention; intervention rationale for essential elements; intervention materials and details about how to access them; description of the intervention procedures; details of intervention providers; mode of delivery of intervention; location of intervention delivery and key infrastructure; details about the number, duration, intensity and dose of intervention sessions; details of any intervention tailoring; any intervention modifications throughout the study; and details of intervention fidelity assessment, monitoring and level achieved. The TIDieR checklist will help improve the quality of intervention reporting more if it is used not only by study authors, but also journal editors, peer reviewers, ethics committees, and funding agencies. A copy of the checklist is available at: http://www.equatornetwork.org/reporting-guidelines/tidier/.

In summary, incomplete reporting of interventions in physiotherapy studies is an important problem and we endorse the use of the TIDieR checklist as a potential solution. The responsibility for improving intervention reporting extends beyond the authors of individual trials to journal editors and others who can mandate the use of the TIDieR checklist to combat this problem. Mandating the use of the TIDieR checklist would guide authors to describe their interventions better and, consequently, help clinicians to use the interventions and researchers to synthesise and replicate the evidence.

At Physiotherapy Practice and Research, the TIDieR statement will be incorporated into manuscript processing workflow from this issue. When peer reviewers receive the manuscript for review, they will also receive the checklist and be invited to give feedback to the submitting authors about any aspects of the intervention that are missing or unclear. Authors should therefore check their manuscript against the checklist before submission. They are also invited to submit a completed checklist, although this is not compulsory. Submitting authors with questions about the checklist are invited to contact the journal's editorial team at E-mail: j.mcveigh@ulster.ac.uk.

\section{Acknowledgments}

DOI of original article: http://dx.doi.org/10.1016/ j.jphys.2016.02.015.

The Editorial was originally published in Journal of Physiotherapy, 2016, however, this article contains a modified author list and amendments to the description about the journal's incorporation of the TIDieR checklist into its manuscript processing. It is republished with the kind permission of the Australian Physiotherapy Association. For citation purposes, please use the original publication details; J. Physiother. 2016;62: $57-58$.

\section{References}

[1] Herbert R, Jamtvedt G, Birger Hagen K, Mead J. Practical Evidence-based Physiotherapy. 2nd ed. London: Churchill Livingstone; 2012.

[2] Duff JM, Leather H, Walden EO, LaPlant KD, George TJ, Jr. Adequacy of published oncology randomized controlled trials to provide therapeutic details needed for clinical application. J Natl Cancer Inst 2010;102:702-5

[3] Glasziou P, Meats E, Heneghan C, Shepperd S. What is missing from descriptions of treatment in trials and reviews? BMJ 2008;336:1472-4.

[4] Hoffmann TC, Erueti C, Glasziou PP. Poor description of non-pharmacological interventions: Analysis of consecutive sample of randomised trials. BMJ 2013;347:f3755.

[5] Yamato TP, Maher CG, Saragiotto BT, Hoffmann TC, Moseley AM. How completely are physiotherapy interventions described in reports of randomised trials? Physiotherapy 2016. 
[6] Research: Increasing value, reducing waste. Lancet 2014; 383:1-56.

[7] Ioannidis JP, Greenland S, Hlatky MA, Khoury MJ, Macleod MR, Moher D, et al. Increasing value and reducing waste in research design, conduct, and analysis. Lancet 2014;383:16675 .

[8] Hoffmann TC, Glasziou PP, Boutron I, Milne R, Perera R, Moher D, et al. Better reporting of interventions: Template for intervention description and replication (TIDieR) checklist and guide. BMJ 2014;348:g1687.
[9] Schulz KF, Altman DG, Moher D, Group C. CONSORT 2010 statement: Updated guidelines for reporting parallel group randomised trials. BMJ 2010;340:c332.

[10] Chan AW, Tetzlaff JM, Altman DG, Laupacis A, Gotzsche PC, Krleza-Jeric K, et al. SPIRIT 2013 statement: Defining standard protocol items for clinical trials. Ann Intern Med 2013;158:200-7. 